\title{
Transmission of ultrasonic waves at oblique incidence to composite laminates with spring-type interlayer interfaces.
}

\author{
$\operatorname{AUTHOR}(\mathrm{S})$ :
}

Ishii, Yosuke; Biwa, Shiro

\section{CITATION:}

Ishii, Yosuke ... [et al]. Transmission of ultrasonic waves at oblique incidence to composite laminates with spring-type interlayer interfaces.. The Journal of the Acoustical Society of America 2015, 138(5): 2800

ISSUE DATE:

2015-11-05

URL:

http://hdl.handle.net/2433/207703

\section{RIGHT:}

(c) 2015 Acoustical Society of America. This article may be downloaded for personal use only. Any other use requires prior permission of the author and the Acoustical Society of America. The following article appeared in [J. Acoust. Soc Am. 138, 2800 (2015)] and may be found at http://scitation.aip.org/content/asa/journal/jasa/138/5/10.1121/1.4934265. 


\title{
Transmission of ultrasonic waves at oblique incidence to composite laminates with spring-type interlayer interfaces
}

\author{
Yosuke Ishii and Shiro Biwa ${ }^{\text {a) }}$ \\ Department of Aeronautics and Astronautics, Graduate School of Engineering, Kyoto University, Katsura, \\ Nishikyo-ku, Kyoto 615-8540, Japan
}

(Received 2 April 2015; revised 5 August 2015; accepted 28 September 2015; published online 5 November 2015)

\begin{abstract}
The transmission characteristics of ultrasonic waves at oblique incidence to composite laminates are analyzed theoretically by the stiffness matrix method. The analysis takes into account the presence of thin resin-rich regions between adjacent plies as spring-type interfaces with normal and shear stiffnesses. The amplitude transmission coefficient of longitudinal wave through a unidirectional laminate immersed in water is shown to be significantly influenced by the frequency, the interlayer interfacial stiffnesses, and the incident angle. Using Floquet's theorem, the dispersion relation of the infinitely extended laminate structure is calculated and compared to the transmission coefficient of laminates of finite thickness. This reveals that the ranges of frequency and interfacial stiffnesses where the Floquet waves lie in the band-gaps agree well with those where the transmission coefficient of the finite layered structure is relatively small, indicating that the band-gaps appear even in the laminate with a finite number of plies. The amplitude transmission coefficient for an 11-ply carbon-epoxy unidirectional composite laminate is experimentally obtained for various frequencies and incident angles. The low-transmission zones observed in the experimental results, which are due to the critical angle of the quasi-longitudinal wave and the Bragg reflection, are shown to be favorably compared with the theory. (C) 2015 Acoustical Society of America.
\end{abstract}

[http://dx.doi.org/10.1121/1.4934265]

$[\mathrm{TK}]$

Pages: $2800-2810$

\section{INTRODUCTION}

Fiber-reinforced composite laminates have been extensively used in aerospace, automotive, marine, and civil engineering since they have the advantages of high specific stiffness, high specific strength, low coefficient of thermal expansion, etc., compared to monolithic materials. Understanding the wave propagation behavior in such structures is important from the viewpoints of the nondestructive characterization of their property as well as their damage diagnosis to ensure the structural safety and reliability.

The ultrasonic wave propagation in composite laminates has been widely studied and various methods have been proposed for the nondestructive evaluation of material properties. ${ }^{1}$ The anisotropic elastic constants of a composite plate can be evaluated by immersing it in fluid and measuring the velocity for three types of transmitted bulk waves at various angles of incidence, i.e., the quasi-longitudinal, fast, and slow quasi-transverse waves. ${ }^{2-6}$ In order to remove the influence of beam refraction at the top and bottom surfaces of specimen, the so-called double through transmission technique was proposed. ${ }^{7,8}$ Extending this procedure, Chu and Rokhlin ${ }^{9}$ evaluated the elastic constants of unidirectionally reinforced plies constituting a cross-ply laminate. Similar measurements for other stacking sequences were performed by Hosten, ${ }^{10}$ and those using the air-coupled transducers were also reported by Hosten et al. ${ }^{11}$ In addition to the velocity measurement, the imaginary part of complex elastic

a)Electronic mail: biwa@kuaero.kyoto-u.ac.jp constants of a unidirectional composite plate can be evaluated as well from the reflected or transmitted amplitude of bulk waves, ${ }^{12-14}$ and the temperature dependence of such properties was investigated by Baudouin and Hosten. ${ }^{15}$ These velocity- or amplitude-based evaluation methods are applicable for sufficiently thick plates where the transmitted bulk modes can be separated in the time domain. Evaluation procedures for the elastic constants of composite laminates for a wider range of their thickness have also been developed, using the amplitude transmission spectrum ${ }^{16}$ or the dispersion curve in the frequency-wavenumber domain. ${ }^{17}$

The above-mentioned studies assumed that all plies of the laminate were perfectly bonded and the effects of interlayer interfaces were ignored. Most resin-based composite laminates such as carbon-epoxy and glass-epoxy laminates, however, have resin-rich regions with a few microns thickness between adjacent plies and such imperfections have significant influence on the mechanical performance of laminated structures; ${ }^{18,19}$ hence, the nondestructive evaluation of the soundness of interlayer interfaces is important. To this end, it is essential to understand the influence of such regions upon the ultrasonic wave propagation behavior.

One way to consider the effect of resin-rich regions on the wave propagation in composite laminates is to model them as a thin resin layer of finite thickness between neighboring plies. For example, Wang and Rokhlin ${ }^{20}$ demonstrated that the resin-rich regions have a significant effect on the reflection characteristics and band-gap behavior of ultrasonic waves at normal incidence to multidirectional composite laminates. Another possible approach is to use 
spring-type interfaces: ${ }^{21-27}$ When performing the wave propagation analysis for adhesive bonds or other imperfect interfaces, the interfacial region much thinner than the wavelength of the ultrasound can be modeled as a springtype interface with finite interfacial normal and shear stiffnesses. On the basis of this idea, the influence of random deviations in the interlayer interfacial normal stiffness or the ply wave velocity on the propagation behavior of the longitudinal wave impinging perpendicularly on a composite laminate was analyzed by $\mathrm{Lu}$ and Achenbach $^{28}$ and $\mathrm{Lu}^{29}$ Ishii and $\mathrm{Biwa}^{30,31}$ studied the influence of interfacial normal stiffness on the wave propagation and band-gap behavior theoretically, and showed that the interfacial normal stiffness can be evaluated from the extremal frequencies of amplitude reflection spectrum. The above-mentioned works ${ }^{20,28-31}$ are, however, limited to the simplest case of the normal incidence of longitudinal wave. The effect of resin-rich regions on the wave propagation behavior of composite laminates for arbitrary angles of incidence remains as a subject to be explored in further depth.

In the present study, the ultrasonic wave propagation in composite laminates with spring-type interlayer interfaces at oblique incidence is analyzed theoretically by using the stiffness-matrix approach. ${ }^{32,33}$ In particular, the influence of the frequency, the interlayer interfacial stiffnesses, and the angle of incidence on the wave transmission and band-gap behavior is elucidated. The present analysis is, however, focused on unidirectional lay-ups for which the interlayer interfaces are solely responsible for the band-gap formation. This paper is constructed as follows: In Sec. II, the formulation of the stiffness-matrix method is briefly summarized and the amplitude transmission coefficient of a unidirectional composite laminate immersed in water is calculated for various frequencies, interfacial normal and shear stiffnesses, and angles of incidence. Using Floquet's theo$\mathrm{rem}^{34-36}$ combined with the stiffness matrix, the dispersion relation of the infinitely extended laminate structure is calculated and compared to the transmission characteristics of laminates of finite thickness in Sec. III. Such a comparison between finite and infinite laminated structures has been considered by Wang and Rokhlin ${ }^{20}$ for multidirectional composite laminates. In the present study, the generation behavior of band-gaps of Floquet waves is examined for arbitrary propagation directions in the presence of spring-type interlayer interfaces in the unidirectional laminate. Comparison between experimental and theoretical transmission coefficients for an 11-ply carbon-epoxy composite laminate is shown in Sec. IV, and the conclusion of this study is summarized in Sec. V.

\section{ANALYSIS OF WAVE PROPAGATION BEHAVIOR IN A UNIDIRECTIONAL COMPOSITE LAMINATE WITH SPRING-TYPE INTERLAYER INTERFACES AT OBLIQUE INCIDENCE}

\section{A. Computation of reflection/transmission coefficient by stiffness matrix method}

Consider a unidirectional composite laminate consisting of $N$ anisotropic elastic plies and $(N-1)$ spring-type interlayer interfaces as shown in Fig. 1. If the harmonic plane longitudinal wave with unit amplitude impinges obliquely on the laminate immersed in ideal fluid from $x_{3}>Z_{0}$ with the direction determined by the angle of incidence $\theta$ as well as the plane of propagation $\varphi$, then the displacements of incident, reflected, and transmitted waves can be written as

$$
\begin{aligned}
\mathbf{u}^{\text {inc }}= & \left(\begin{array}{c}
\sin \theta \sin \phi \\
-\sin \theta \cos \phi \\
-\cos \theta
\end{array}\right) \\
& \times e^{i\left[k_{\mathrm{f}}\left\{x_{1} \sin \theta \sin \phi-x_{2} \sin \theta \cos \phi-\left(x_{3}-Z_{0}\right) \cos \theta\right\}-\omega t\right]} \\
& \left(x_{3}>Z_{0}\right), \\
\mathbf{u}^{\text {ref }}= & R\left(\begin{array}{c}
\sin \theta \sin \phi \\
-\sin \theta \cos \phi \\
\cos \theta
\end{array}\right) \\
& \times e^{i\left[k_{\mathrm{f}}\left\{x_{1} \sin \theta \sin \phi-x_{2} \sin \theta \cos \phi+\left(x_{3}-Z_{0}\right) \cos \theta\right\}-\omega t\right]} \\
& \left(x_{3}>Z_{0}\right), \\
\mathbf{u}^{\text {tra }}= & T\left(\begin{array}{c}
\sin \theta \sin \phi \\
-\sin \theta \cos \phi \\
-\cos \theta
\end{array}\right) \\
& \times e^{i\left[k_{\mathrm{f}}\left\{x_{1} \sin \theta \sin \phi-x_{2} \sin \theta \cos \phi-\left(x_{3}-Z_{0}\right) \cos \theta\right\}-\omega t\right]} \\
& \left(x_{3}<Z_{N}\right),
\end{aligned}
$$

respectively, where $\omega(=2 \pi f)$ is the angular frequency and $k_{\mathrm{f}}$ is the wavenumber in the fluid. The complex reflection and transmission coefficients $R$ and $T$ can be calculated by the stiffness matrix method ${ }^{32,33}$ as

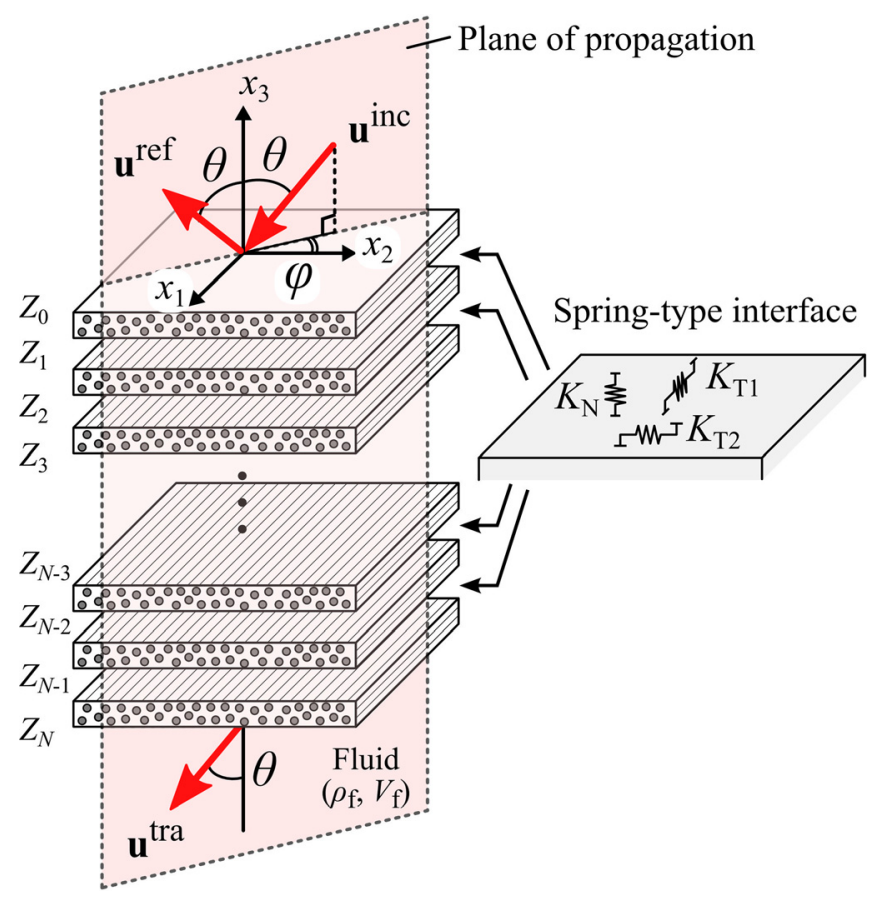

FIG. 1. (Color online) A unidirectional composite laminate with spring-type interlayer interfaces immersed in ideal fluid. 


$$
\begin{aligned}
R & =\frac{\left(\gamma+S_{33}^{\mathrm{G}}\right)\left(\gamma+S_{66}^{\mathrm{G}}\right)-S_{36}^{\mathrm{G}} S_{63}^{\mathrm{G}}}{\left(\gamma-S_{33}^{\mathrm{G}}\right)\left(\gamma+S_{66}^{\mathrm{G}}\right)+S_{36}^{\mathrm{G}} S_{63}^{\mathrm{G}}}, \\
T & =\frac{-\gamma 2 S_{63}^{\mathrm{G}}}{\left(\gamma-S_{33}^{\mathrm{G}}\right)\left(\gamma+S_{66}^{\mathrm{G}}\right)+S_{36}^{\mathrm{G}} S_{63}^{\mathrm{G}}} e^{-i k_{\mathrm{f}} H \cos \theta}, \\
\gamma & =\frac{\cos \theta}{i \rho_{\mathrm{f}} V_{\mathrm{f}} \omega},
\end{aligned}
$$

where $\rho_{\mathrm{f}}$ and $V_{\mathrm{f}}=\omega / k_{\mathrm{f}}$ are the density and wave speed of the surrounding fluid and $H=Z_{0}-Z_{N}$ is the laminate thickness. In the above expression, $S_{i j}{ }^{\mathrm{G}}$ are the components of the inverse of $6 \times 6$ global stiffness matrix which is obtained by applying the recursive algorithm ${ }^{32}$ to all of the local stiffness matrices of the ply $\mathbf{K}_{m}^{\text {ply }}(m=1,2, \ldots, N)$ as well as the interlayer interface $\mathbf{K}_{m}^{\mathrm{sp}}(m=1,2, \ldots, N-1)$ defined as

$$
\begin{gathered}
\left(\begin{array}{c}
\left.\boldsymbol{\sigma}\right|_{x_{3}=Z_{m-1,-}} \\
\left.\boldsymbol{\sigma}\right|_{x_{3}=Z_{m,+}}
\end{array}\right)=\mathbf{K}_{m}^{\mathrm{ply}}\left(\begin{array}{c}
\left.\mathbf{u}\right|_{x_{3}=Z_{m-1,-}} \\
\left.\mathbf{u}\right|_{x_{3}=Z_{m,+}}
\end{array}\right), \\
\left(\begin{array}{c}
\left.\boldsymbol{\sigma}\right|_{x_{3}=Z_{m,+}} \\
\left.\boldsymbol{\sigma}\right|_{x_{3}=Z_{m,-}}
\end{array}\right)=\mathbf{K}_{m}^{\mathrm{sp}}\left(\begin{array}{c}
\left.\mathbf{u}\right|_{x_{3}=Z_{m,+}} \\
\left.\mathbf{u}\right|_{x_{3}=Z_{m,-}}
\end{array}\right), \\
\boldsymbol{\sigma}=\left(\begin{array}{c}
\sigma_{13} \\
\sigma_{23} \\
\sigma_{33}
\end{array}\right), \quad \mathbf{u}=\left(\begin{array}{c}
u_{1} \\
u_{2} \\
u_{3}
\end{array}\right),
\end{gathered}
$$

where $x_{3}=Z_{m}(m=1,2, \ldots, N-1)$ correspond to the location of interlayer interfaces, and the subscript "+" ("-") denotes that the corresponding coordinate is approached from the positive (negative) $x_{3}$ side. In the present analysis, the matrices $\mathbf{K}_{m}^{\mathrm{ply}}$ and $\mathbf{K}_{m}^{\mathrm{sp}}$ are assumed to be the same for all plies and interfaces. The latter can be calculated by using the interfacial normal and shear stiffnesses, $K_{\mathrm{N}}, K_{\mathrm{T} 1}$, and $K_{\mathrm{T} 2}$, as

$$
\mathbf{K}_{m}^{\mathrm{sp}}=\left[\begin{array}{cccccc}
K_{\mathrm{T} 1} & 0 & 0 & -K_{\mathrm{T} 1} & 0 & 0 \\
0 & K_{\mathrm{T} 2} & 0 & 0 & -K_{\mathrm{T} 2} & 0 \\
0 & 0 & K_{\mathrm{N}} & 0 & 0 & -K_{\mathrm{N}} \\
K_{\mathrm{T} 1} & 0 & 0 & -K_{\mathrm{T} 1} & 0 & 0 \\
0 & K_{\mathrm{T} 2} & 0 & 0 & -K_{\mathrm{T} 2} & 0 \\
0 & 0 & K_{\mathrm{N}} & 0 & 0 & -K_{\mathrm{N}}
\end{array}\right]
$$

The above expression is valid to model the interlayer imperfections of unidirectional composite laminates where the fiber direction of all plies is identical with $x_{1}$ (or $x_{2}$ ) direction. Other stacking sequences such as angle-ply, cross-ply, or quasi-isotropic lay-up can be modeled by including additional non-zero terms which express the coupling effects ${ }^{24}$ in Eq. (6) and allow the matrix components to vary for different interfaces. For the details of the calculation of $\mathbf{K}_{m}^{\mathrm{ply}}$ and the recursive algorithm mentioned above, refer to Refs. 32 and 33. It should be noted that the stiffness matrix approach can compute the reflection and transmission coefficients more stably than the transfer matrix approach ${ }^{37-40}$ which encounters the numerical instability issues for high frequency or large ply thickness.

\section{B. Influence of interlayer interfacial stiffness and angle of incidence on amplitude transmission spectrum}

Using the approach mentioned above, the amplitude transmission spectra of an 11-ply unidirectional composite laminate immersed in water are calculated for various interlayer interfacial stiffnesses and angles of incidence with the material properties in Table I, and depicted in Figs. 2 and 3. The unidirectionally reinforced plies are modeled as transversely isotropic elastic media and stacked so that the fiber direction is identical with the $x_{1}$ direction for all plies. Note that the imaginary parts of ply stiffness shown in Table I are neglected throughout the numerical analysis in Secs. II and III, but are taken into account in the comparison between experiment and theory in Sec. IV. The angle $\varphi$ which represents the deviation of the plane of propagation from the isotropic plane of ply $\left(x_{2}-x_{3}\right.$ plane $)$ is fixed as $45^{\circ}$, and for simplicity of analysis, it is assumed that the interfacial shear stiffness is orientation-independent, i.e., $K_{\mathrm{T} 1}=K_{\mathrm{T} 2} \equiv K_{\mathrm{T}}$. The horizontal axis in Figs. 2 and 3, whose range corresponds to approximately $0 \leq f \leq 16 \mathrm{MHz}$, is normalized by the ply thickness and the longitudinal wave velocity of the ply in the $x_{3}$ direction, while the vertical ones, whose ranges correspond to $0.08 \leq K_{\mathrm{N}} \leq 8 \mathrm{MPa} / \mathrm{nm}$ and $0.04 \leq K_{\mathrm{T}} \leq 4 \mathrm{MPa} / \mathrm{nm}$, respectively, are normalized by the ply thickness and stiffness.

It is seen in Figs. 2 and 3 that the transmission coefficient is influenced by not only the frequency and interfacial stiffnesses but also the angle of incidence. This is because the number of propagating bulk modes existing in the laminate changes according to the latter parameter. Wang and Rokhlin ${ }^{20}$ examined this variation by invoking the critical angles of Floquet waves for multidirectional laminates. Since the present analysis focuses on unidirectional laminates, the critical angles of Floquet waves can be reasonably approximated by those of bulk waves in the ply. In Fig. 4, the critical angles of the quasi-longitudinal and two quasitransverse waves at an interface between water and the ply are shown as a function of the plane of propagation $\varphi$. Because of the anisotropic nature of the ply, the critical angles are dependent on $\varphi$ and they take $16^{\circ}, 36^{\circ}$, and $56^{\circ}$ at $\varphi=45^{\circ}$. In the case of normal incidence [Figs. 2(a) and $3(\mathrm{a})$, only the pure longitudinal mode propagates in the laminate in the direction normal to the interfaces, so the transmission coefficient is influenced only by the normal component of the interlayer interfacial stiffness. It is also seen that the transmission coefficient oscillates with the frequency due to the finite number of plies. ${ }^{30}$ As the angle of

TABLE I. Material properties of the composite laminate and the surrounding fluid (water).

\begin{tabular}{lc}
\hline \hline Stacking sequence of laminate & {$[0]_{11}$} \\
Complex elastic constants & \\
of ply (transverse isotropy & \\
with $x_{1}$ as fiber direction is assumed.) & \\
$C_{11}$ & $81.5-2.90 i \mathrm{GPa}$ \\
$C_{13}$ & $4.9-0.33 i \mathrm{GPa}$ \\
$C_{33}$ & $15.2-0.29 i \mathrm{GPa}$ \\
$C_{44}$ & $3.2-0.14 i \mathrm{GPa}$ \\
$C_{66}$ & $6.9-0.24 i \mathrm{GPa}$ \\
Density of ply $\rho$ & $1.5 \times 10^{3} \mathrm{~kg} / \mathrm{m}^{3}$ \\
Thickness of ply $h$ & $0.19 \mathrm{~mm}$ \\
Density of fluid $\rho_{\mathrm{f}}$ & $1.0 \times 10^{3} \mathrm{~kg} / \mathrm{m}^{3}$ \\
Wave speed in fluid $V_{\mathrm{f}}$ & $1.5 \times 10^{3} \mathrm{~m} / \mathrm{s}$ \\
\hline
\end{tabular}




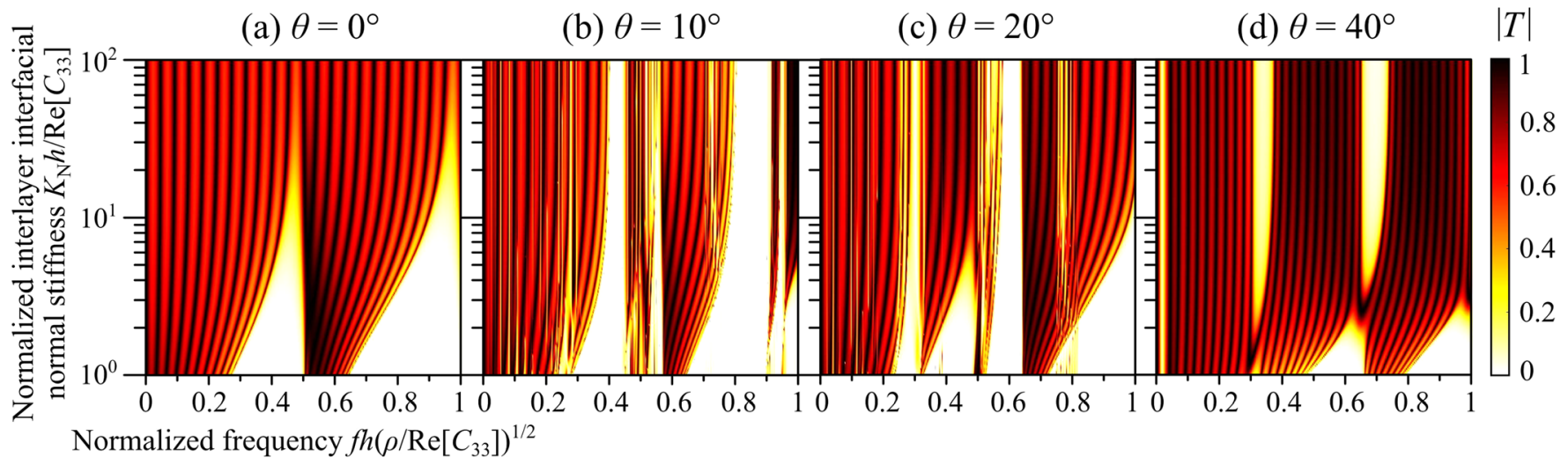

FIG. 2. (Color online) The variation of amplitude transmission coefficient with the normalized frequency and the normalized interlayer interfacial normal stiffness for four different angles of incidence with fixed interfacial shear stiffness $K_{\mathrm{T}}=0.04 \mathrm{MPa} / \mathrm{nm}\left(K_{\mathrm{T}} h / \operatorname{Re}\left[C_{66}\right]=1\right)$ and plane of propagation $\varphi=45^{\circ}$.

incidence increases from zero [Figs. 2(b) and 3(b)], two other bulk modes come to propagate in the plies. In this case, the quasi-longitudinal as well as two quasi-transverse modes in general have the displacement components parallel to the interface. Hence, the interfacial shear stiffness is also involved and the transmission coefficient exhibits the more complicated behavior than the normal incidence case. In Figs. 2(c) and $3(\mathrm{c})$, where the angle of incidence is above the critical angle of quasi-longitudinal wave, the number of bulk modes in the plies reduces to two, but the transmission characteristics remain complex. When the angle of incidence further increases so as to exceed the next critical angle of fast quasitransverse wave, only the slow quasi-transverse mode can propagate in the laminate in the thickness direction, and the patterns of the transmission coefficient shown in Figs. 2(d) and 3(d) become simpler as in the case of normal incidence where the transmission characteristics are governed by a single bulk mode.

Furthermore, it is recognized that there are certain frequency ranges in Figs. 2 and 3 where the transmission coefficient falls to a low level. For example, two frequency bands of vanishingly small transmission coefficient are seen in Fig. 2(a) spanning approximately for $0.28 \leq f h\left(\rho / \operatorname{Re}\left[C_{33}\right]\right)^{1 / 2} \leq 0.50$ and $0.65 \leq f h\left(\rho / \operatorname{Re}\left[C_{33}\right]\right)^{1 / 2} \leq 1$ when $K_{\mathrm{N}} h / \operatorname{Re}\left[C_{33}\right]=1$. Their location and bandwidth are, as a general trend, influenced by the interfacial stiffnesses and the angle of incidence.

\section{DISPERSION RELATION OF INFINITELY PERIODIC STRUCTURE}

In this section, the generation of low-transmission frequency ranges found Sec. II B is investigated in more detail based on the dispersion relation of the infinitely periodic structure.

\section{A. Computation of Floquet wavenumber}

Consider a periodic structure shown in Fig. 5, which has an infinite number of unit-cells each consisting of a unidirectional ply and a spring-type interface. The stiffness matrix for a unit-cell $\mathbf{K}$ is given as

$$
\left(\begin{array}{c}
\left.\boldsymbol{\sigma}\right|_{x_{3}=Z_{m-1,+}} \\
\left.\boldsymbol{\sigma}\right|_{x_{3}=Z_{m,+}}
\end{array}\right)=\mathbf{K}\left(\begin{array}{c}
\left.\mathbf{u}\right|_{x_{3}=Z_{m-1,+}} \\
\left.\mathbf{u}\right|_{x_{3}=Z_{m,+}}
\end{array}\right), \quad \mathbf{K} \equiv\left[\begin{array}{ll}
\mathbf{K}_{11} & \mathbf{K}_{12} \\
\mathbf{K}_{21} & \mathbf{K}_{22}
\end{array}\right],
$$

where $\mathbf{K}_{i j}(i, j=1,2)$ represent $3 \times 3$ matrices. For such a periodic system, Floquet's theorem yields the so-called periodic relation

$$
\left(\begin{array}{c}
\left.\boldsymbol{\sigma}\right|_{x_{3}=Z_{m-1,+}} \\
\left.\mathbf{u}\right|_{x_{3}=Z_{m-1,+}}
\end{array}\right)=\exp \left(i \zeta h_{\mathbf{u}}\right)\left(\begin{array}{c}
\left.\boldsymbol{\sigma}\right|_{x_{3}=Z_{m,+}} \\
\left.\mathbf{u}\right|_{x_{3}=Z_{m,+}}
\end{array}\right),
$$

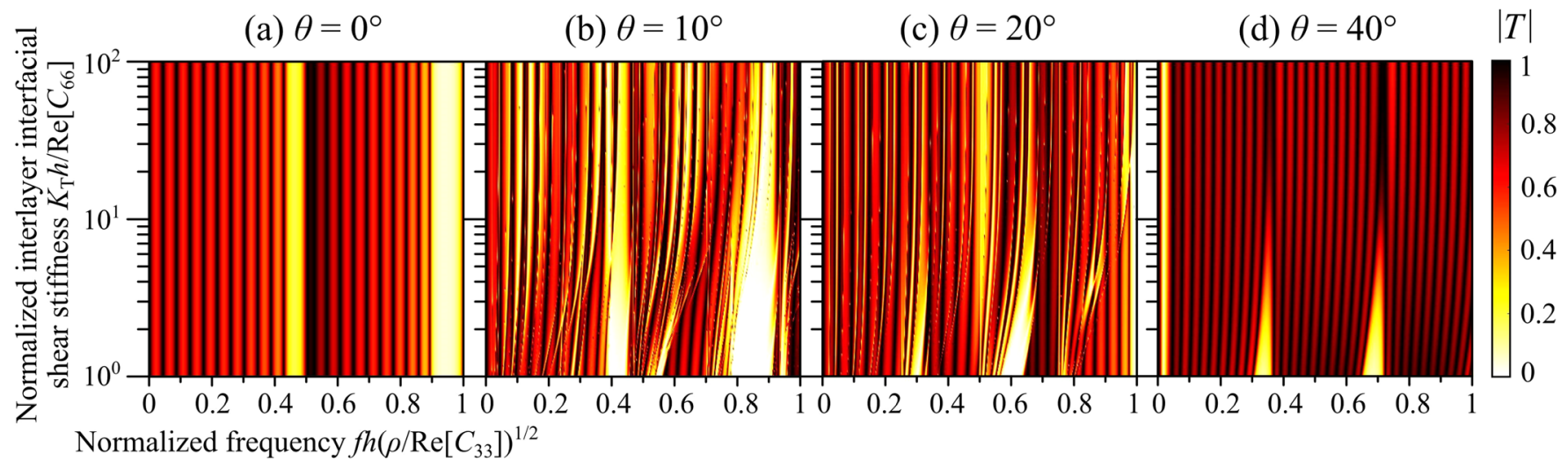

FIG. 3. (Color online) The variation of amplitude transmission coefficient with the normalized frequency and the normalized interlayer interfacial shear stiffness for four different angles of incidence with fixed interfacial normal stiffness $K_{\mathrm{N}}=0.8 \mathrm{MPa} / \mathrm{nm}\left(K_{\mathrm{N}} h / \operatorname{Re}\left[C_{33}\right]=10\right)$ and plane of propagation $\varphi=45^{\circ}$. 


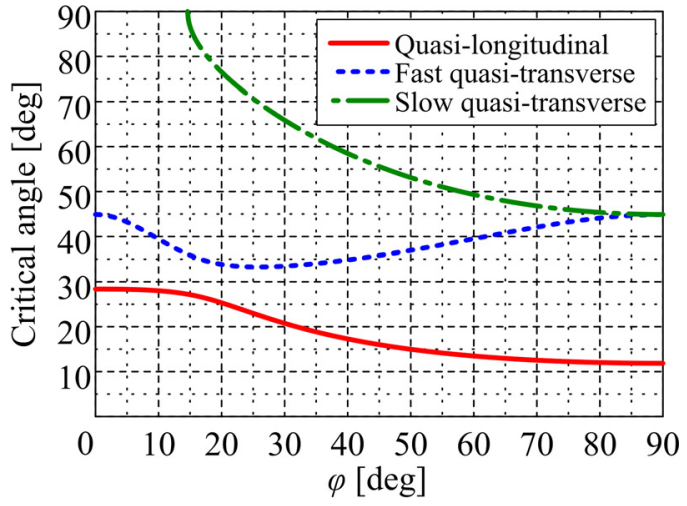

FIG. 4. (Color online) The critical angle of three bulk modes at the waterply interface.

where $\zeta$ is the $x_{3}$ component of the Floquet wavenumber ${ }^{34-36}$ and $h_{\mathrm{u}}=Z_{m-1,+}-Z_{m,+}(=h)$ is the thickness of unit-cell (the theorem is also referred to as the Bloch theorem but the nomenclature commonly used in many studies for composite laminates $^{34-36}$ is followed here). From Eqs. (7) and (8), the characteristic equation for the Floquet wave in the infinitely periodic structure can be written as

$$
A_{3} \cos \left(3 \zeta h_{\mathrm{u}}\right)+A_{2} \cos \left(2 \zeta h_{\mathrm{u}}\right)+A_{1} \cos \left(\zeta h_{\mathrm{u}}\right)+A_{0}=0,
$$

where

$$
\begin{aligned}
A_{3}= & \operatorname{det}\left(\mathbf{K}_{21}\right), \\
A_{2}= & \frac{1}{2}\left\{\operatorname{det}\left(\mathbf{K}_{22}-\mathbf{K}_{11}+\mathbf{K}_{21}\right)\right. \\
& \left.+\operatorname{det}\left(\mathbf{K}_{22}-\mathbf{K}_{11}-\mathbf{K}_{21}\right)\right\}-\operatorname{det}\left(\mathbf{K}_{22}-\mathbf{K}_{11}\right),
\end{aligned}
$$

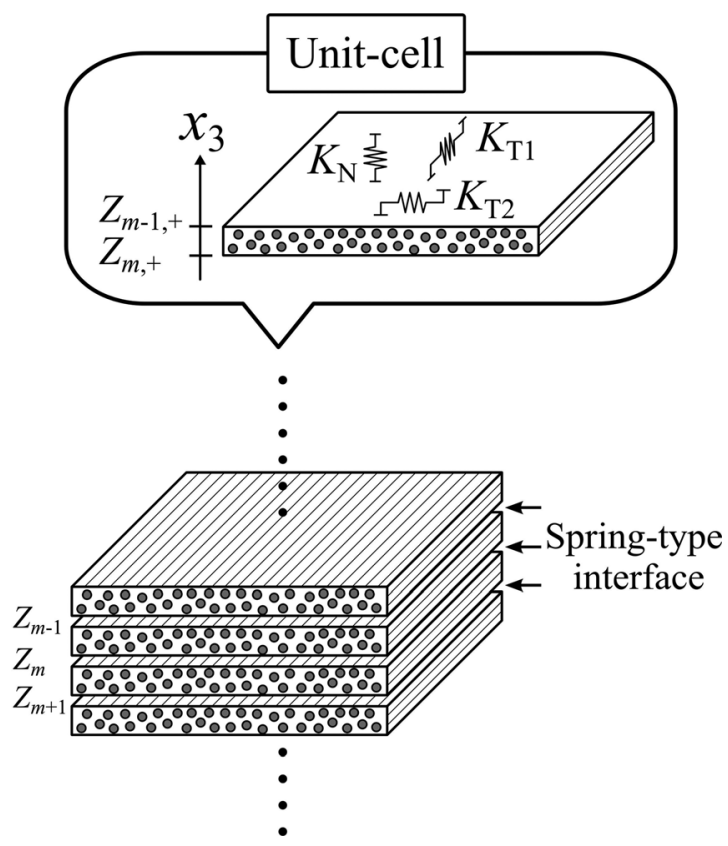

FIG. 5. An infinitely laminated composite and its unit-cell.

$$
\begin{aligned}
A_{1}= & \frac{1}{2}\left\{\operatorname{det}\left(\mathbf{K}_{22}-\mathbf{K}_{11}+\mathbf{K}_{21}\right)+\operatorname{det}\left(\mathbf{K}_{22}-\mathbf{K}_{11}+\mathbf{K}_{12}\right)\right. \\
& \left.+\operatorname{det}\left(\mathbf{K}_{21}-\mathbf{K}_{12}\right)\right\}-2 \operatorname{det}\left(\mathbf{K}_{21}\right), \\
A_{0}= & \frac{1}{4}\left\{\operatorname{det}\left(\mathbf{K}_{22}-\mathbf{K}_{11}+\mathbf{K}_{12}-\mathbf{K}_{21}\right)\right. \\
& \left.+\operatorname{det}\left(\mathbf{K}_{22}-\mathbf{K}_{11}-\mathbf{K}_{12}+\mathbf{K}_{21}\right)\right\}-A_{2} .
\end{aligned}
$$

Equation (9) has six solutions for $\zeta$ in the range of $-\pi / h_{\mathrm{u}}$ $\leq \operatorname{Re}[\zeta] \leq \pi / h_{\mathrm{u}}$, which satisfy the relation $\zeta_{1}=-\zeta_{4}, \zeta_{2}=-\zeta_{5}$, and $\zeta_{3}=-\zeta_{6}$, i.e., they correspond to three pairs of Floquet waves propagating in the opposite $x_{3}$ directions. This characteristic equation was derived by Wang and Rokhlin, ${ }^{36}$ but the correct expressions of its coefficients have been rederived here as shown in Eqs. (10)-(13). From Eq. (9), $\zeta$ can be given as

$$
\zeta=\frac{2 m \pi+\arg (X)}{h_{\mathrm{u}}}-i \frac{\ln |X|}{h_{\mathrm{u}}},
$$

where $m$ is an integer, $\arg (\bullet)$ and $|\bullet|$ represent the argument and modulus of a complex number, and $X\left(=\exp \left(i \zeta h_{\mathrm{u}}\right)\right)$ is a root of

$$
X^{2}-\eta X+1=0
$$

where $\eta$ is a root of the following cubic equation:

$$
A_{3} \eta^{3}+A_{2} \eta^{2}+\left(A_{1}-3 A_{3}\right) \eta+2\left(A_{0}-A_{2}\right)=0 .
$$

In what follows, the Floquet waves propagating in the positive $x_{3}$ direction are only considered, whose wavenumbers are denoted by $\zeta_{1}, \zeta_{2}$, and $\zeta_{3}$. The relation between the $x_{3}$ component of the normalized Floquet wavenumbers and the normalized frequency for the laminate parameters given in Table I (imaginary parts of ply stiffness are neglected as mentioned in Sec. II B) is illustrated in Fig. 6 for different propagation directions expressed in terms of the corresponding incident angle from water. The real part of the wavenumbers is drawn with the so-called extended zone scheme, ${ }^{34}$ where the integer $m$ in Eq. (14) is determined so that the dispersion curve becomes continuous. The angle for plane of propagation $\varphi$ is fixed again as $45^{\circ}$ and the normalized interlayer interfacial stiffnesses as $K_{\mathrm{N}} h / \operatorname{Re}\left[C_{33}\right]=12.5$ and $K_{\mathrm{T}} h /$ $\operatorname{Re}\left[C_{66}\right]=2.8$, which correspond to $K_{\mathrm{N}}=1 \mathrm{MPa} / \mathrm{nm}$ and $K_{\mathrm{T}}=0.1 \mathrm{MPa} / \mathrm{nm}$, respectively.

The Floquet wave is a linear combination of three types of classical plane waves ${ }^{35}$ which propagate in the ply, i.e., one quasi-longitudinal and two quasi-transverse waves. With the stacking sequence concerned here, however, each Floquet wave is dominated by one of them. In the case of normal incidence or in the limiting case of $K_{\mathrm{N}}=K_{\mathrm{T}}=\infty$ (perfect bonding), each Floquet wave consists of a single classical plane wave. For this reason, three Floquet waves in Fig. 6 are denoted by referring to the corresponding dominant plane wave modes.

It is seen in Fig. 6 that when the frequency increases from zero, the real parts of the Floquet wavenumber also 
(a) $\theta=0^{\circ}$

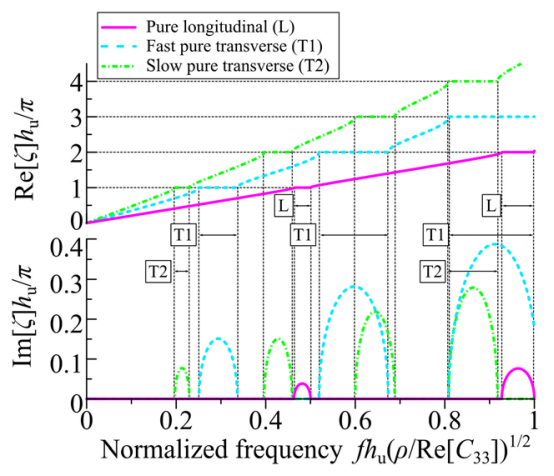

(c) $\theta=20^{\circ}$

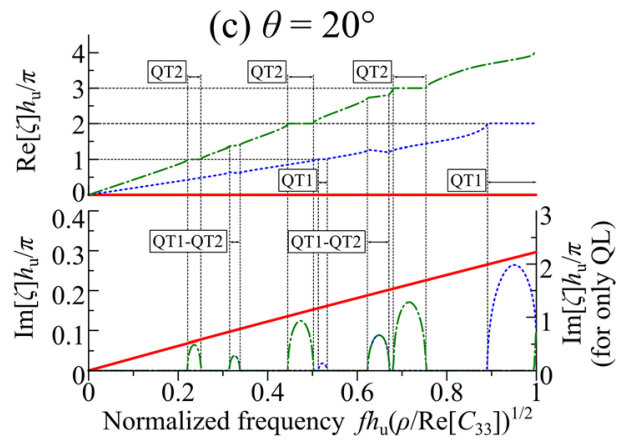

(b) $\theta=10^{\circ}$

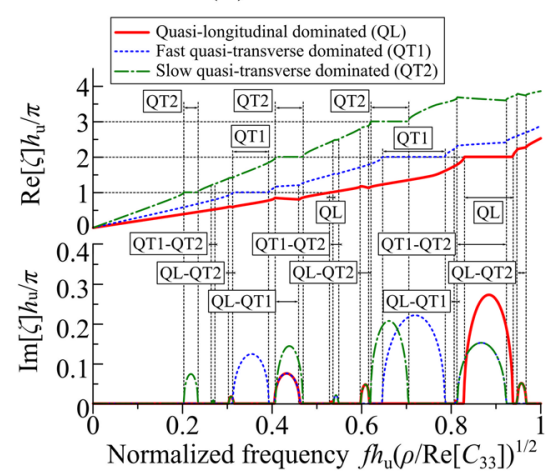

(d) $\theta=40^{\circ}$

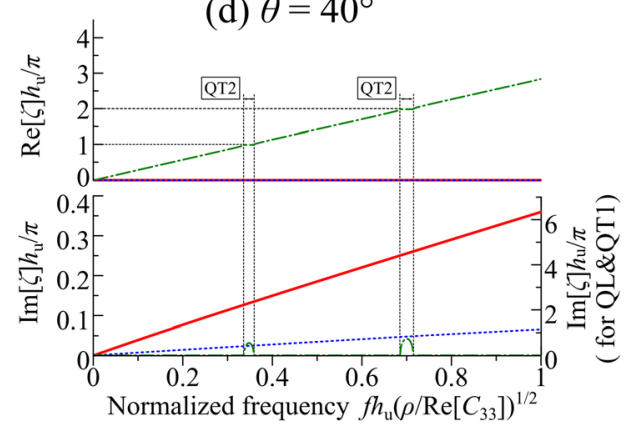

FIG. 6. (Color online) The dispersion relation of Floquet waves for four different corresponding angles of incidence from water with fixed interlayer interfacial normal stiffness $K_{\mathrm{N}}=1 \mathrm{MPa} / \mathrm{nm}$, shear stiffness $K_{\mathrm{T}}=0.1 \mathrm{MPa} / \mathrm{nm}$, and plane of propagation $\varphi=45^{\circ}$. increase from zero, and in certain frequency ranges they possess non-zero imaginary parts. These ranges are the so-called frequency band-gaps (or stopping bands), which indicate that the corresponding mode cannot propagate in the periodic structure since the Bragg reflection occurs. Such band-gaps appear when the following condition is satisfied:

$$
\operatorname{Re}\left[\zeta_{\alpha}\right] h_{\mathrm{u}} / \pi+\operatorname{Re}\left[\zeta_{\beta}\right] h_{\mathrm{u}} / \pi=2 n,(\alpha, \beta=1,2,3),
$$

where $n$ stands for an integer. The derivation of the above relation is given in the Appendix. When $\alpha=\beta$ in Eq. (17), the Bragg reflection occurs with a single Floquet wave, which results in the single band-gaps indicated in Fig. 6 by "L," “T1," “T2," "QL," "QT1," and "QT2." On the other hand, when $\alpha \neq \beta$, two Floquet waves suffer the Bragg reflection in combination and the resulting double band-gaps are indicated by "QL-QT1," "QL-QT2," and "QT1-QT2."

When $\theta=0^{\circ}$ [Fig. 6(a)], three Floquet waves are identical with the pure longitudinal (L) and transverse (T1 and T2) modes as mentioned above. Since such modes do not couple with one another, i.e., no mode conversion occurs at the interlayer interfaces, there exist only single band-gaps in Fig. 6(a). When $\theta=10^{\circ}$ [Fig. 6(b)], the Floquet waves consist of different classical plane waves which undergo the mode conversion at every interface, so not only the single but also double band-gaps are generated. This is also true for $\theta=20^{\circ}$ [Fig. 6(c)] except that the Floquet wave dominated by the quasi-longitudinal mode, which propagates in the direction parallel to the interfaces, has no influence on the generation of band-gaps. When $\theta=40^{\circ}$ [Fig. $6(d)$, the wavenumbers of two faster Floquet waves become pure imaginary, and the band structure is determined only by the remaining wave dominated by the slow quasi-transverse mode.

\section{B. Comparison with transmission characteristics of finite layered structure}

Using the stiffness matrix method again, the amplitude transmission spectra of the immersed composite laminate are calculated for different number of plies with fixed interfacial stiffnesses $K_{\mathrm{N}}=1 \mathrm{MPa} / \mathrm{nm}$ and $K_{\mathrm{T}}=0.1 \mathrm{MPa} / \mathrm{nm}$, angle of incidence $\theta=10^{\circ}$, and plane of propagation $\varphi=45^{\circ}$, and shown in Fig. 7. The low-transmission frequency ranges centered at around $f h\left(\rho / \operatorname{Re}\left[C_{33}\right]\right)^{1 / 2}=0.43$ and 0.87 become more distinct as the number of plies increases, and such regions are in good agreement with the band-gaps of the corresponding infinitely layered structure in Fig. 6(b). This indicates that the band-gaps appear even in the finite layered laminate and becomes remarkable with the increasing number of plies. It is also found that the band-gaps of the Floquet waves dominated by quasi-transverse modes seen in Fig. 6(b) do not form clear low-transmission ranges in Fig. 7.

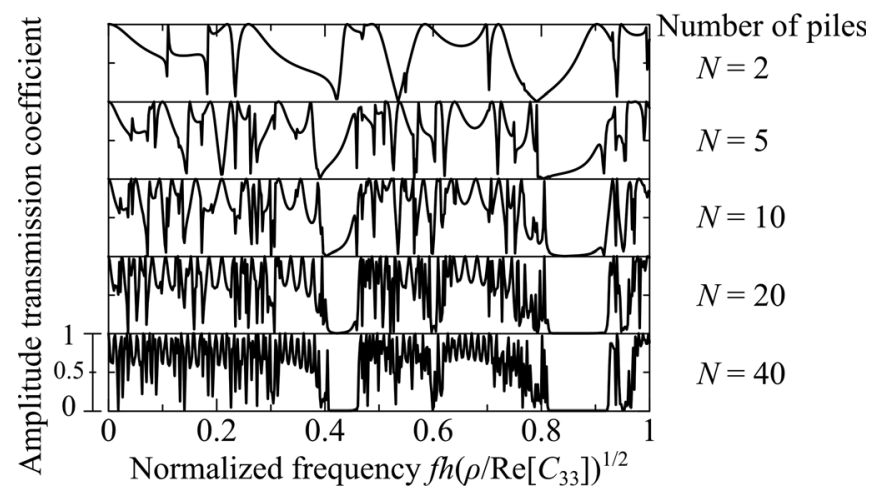

FIG. 7. The variation of the amplitude transmission spectrum with the number of plies for fixed interlayer interfacial normal stiffness $K_{\mathrm{N}}=1 \mathrm{MPa} / \mathrm{nm}$, shear stiffness $K_{\mathrm{T}}=0.1 \mathrm{MPa} / \mathrm{nm}$, angle of incidence $\theta=10^{\circ}$, and plane of propagation $\varphi=45^{\circ}$. 


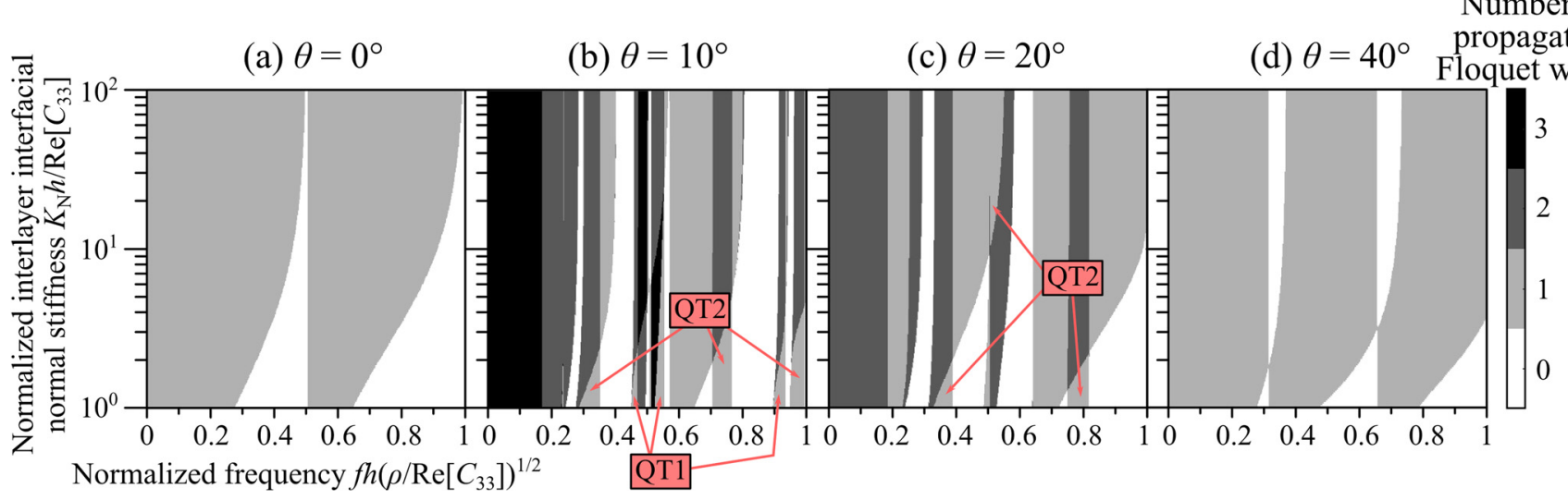

FIG. 8. (Color online) The variation of the number of propagating Floquet waves with the normalized frequency and the normalized interfacial normal stiffness for four different corresponding angles of incidence from water with fixed $K_{\mathrm{T}}=0.04 \mathrm{MPa} / \mathrm{nm}\left(K_{\mathrm{T}} h / \operatorname{Re}\left[C_{66}\right]=1\right)$ and plane of propagation $\varphi=45^{\circ}$.

This is because the wave field in the finite layered laminate is mainly governed by the quasi-longitudinal mode; namely, even if the Floquet waves dominated by the quasi-transverse modes lie in the band-gaps, the ultrasonic wave can transmit through the laminate as the quasi-longitudinal mode.

With the same parameters used for the computation of Figs. 2 and 3, the number of propagating Floquet waves is calculated and shown in Figs. 8 and 9 as functions of the normalized frequency, the normalized interlayer interfacial stiffnesses, and the corresponding angle of incidence from water. Note that for $\theta=0^{\circ}$ in Figs. 8(a) and 9(a), only the pure longitudinal mode is counted in order to compare with Figs. 2(a) and 3(a) as it is the only wave that can couple with water. It is seen that if the number of propagating Floquet waves becomes zeros (white zones) in Figs. 8 and 9, then the transmission coefficients drops to a low level in Figs. 2 and 3 , which reconfirms the results mentioned in the last paragraph. Its converse is not necessarily true, however, particularly in the cases of $\theta=10^{\circ}$ and $20^{\circ}$. In the ranges of approximately $0.70 \leq f h\left(\rho / \operatorname{Re}\left[C_{33}\right]\right)^{1 / 2} \leq 0.76$ and $1 \leq K_{\mathrm{N}} h /$ $\operatorname{Re}\left[C_{33}\right] \leq 3$ for $\theta=10^{\circ}$, the Floquet wave dominated by the slow quasi-transverse mode is propagative as indicated by "QT2" in Fig. 8(b), but the transmission coefficient becomes very low in Fig. 2(b). This is, as also mentioned above, because the transmission coefficient is mainly governed by one dominant classical plane wave mode (quasi-longitudinal for $\theta=10^{\circ}$ and fast quasi-transverse for $\theta=20^{\circ}$ ) and not sensitive to whether the other modes can transmit through the laminate or not. In addition to that, when $\theta=40^{\circ}$, there exists a frequency range at around $f h\left(\rho / \operatorname{Re}\left[C_{33}\right]\right)^{1 / 2}=0.02$ where the transmission coefficient takes a relatively small value in Figs. 2(d) and 3(d) but no band-gaps can be seen in Figs. 8(d) and 9(d). This is likely due to the finite thickness of the laminate, i.e., an eigen-vibration of laminate is excited and the reflection becomes remarkable.

Although the plane of propagation has been fixed as $\varphi=45^{\circ}$ in this analysis, the transmission characteristics for other values of $\varphi$ can be explained in the same manner as described above.

\section{COMPARISON WITH EXPERIMENT}

The double-through transmission measurement ${ }^{7,8}$ was carried out for an 11-ply composite laminate with unidirectional stacking sequence. The specimen, which was supplied by Mitsubishi Rayon, Co. Ltd., Japan, was made of TR30 carbon fibers of about $7 \mu \mathrm{m}$ diameter and \#340 epoxy resin, and had the ply thickness of about $0.19 \mathrm{~mm}$. The ultrasonic waves transmitted through the specimen twice were measured for different angles of incidence $\theta$ as well as planes

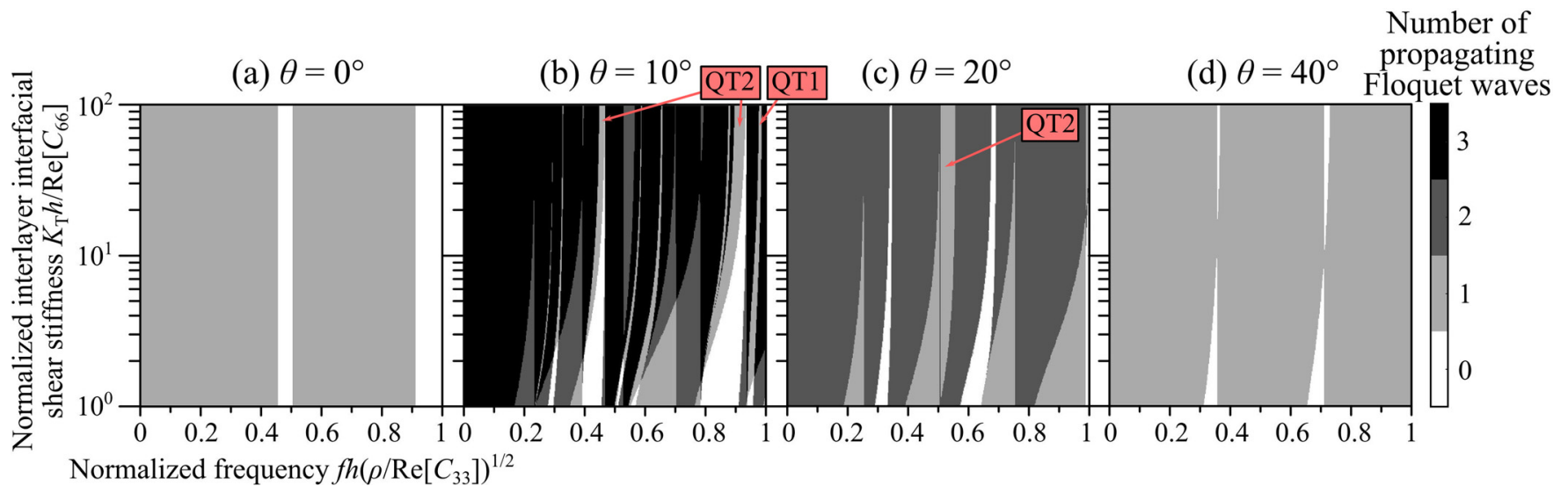

FIG. 9. (Color online) The variation of the number of propagating Floquet waves with the normalized frequency and the normalized interfacial shear stiffness for four different corresponding angles of incidence from water with fixed $K_{\mathrm{N}}=0.8 \mathrm{MPa} / \mathrm{nm}\left(K_{\mathrm{N}} h / \operatorname{Re}\left[C_{33}\right]=10\right)$ and plane of propagation $\varphi=45^{\circ}$. 


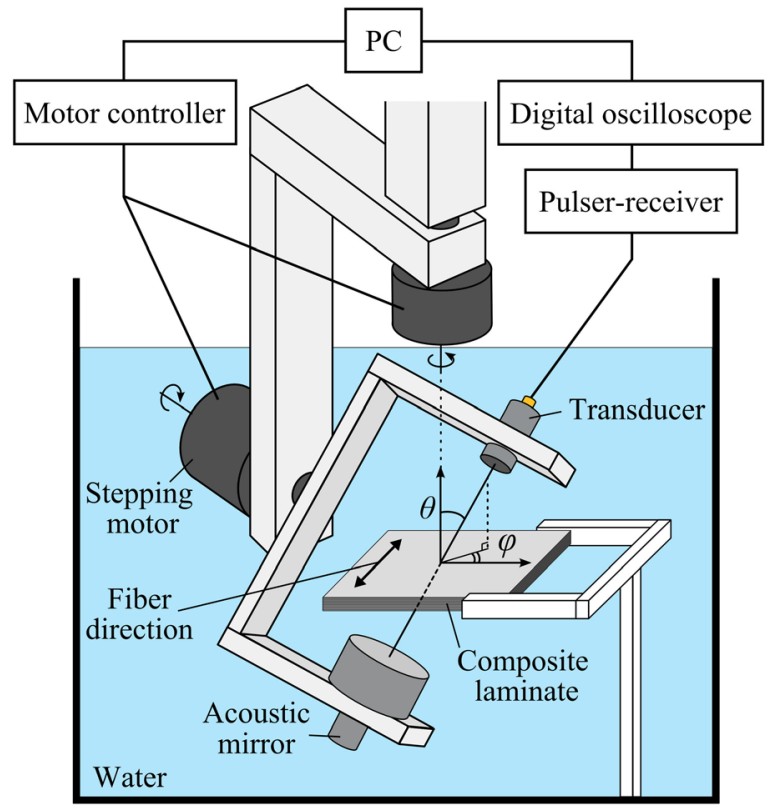

FIG. 10. (Color online) Experimental apparatus.

of propagation $\varphi$ by using a setup shown in Fig. 10, which consists of a personal computer (MATLAB), an Agilent digital oscilloscope DSO5034A, a Panametrics pulser-receiver 5072PR, a motor controller, two five-phase stepping motors with angle resolutions $0.004^{\circ}$ for both $\theta$ and $\varphi$, a piezoelectric broadband transducer with nominal frequency $15 \mathrm{MHz}$ (IT1506R, Insight, Inc., Japan), and an acoustic mirror made of a polished stainless steel disk with $50 \mathrm{~mm}$ diameter and $30 \mathrm{~mm}$ thickness. The signals were measured with a gate length of $10 \mu \mathrm{s}$, which was chosen in order to include not only the first arrival waves but also the following ones due to the multiple reflections inside the specimen but not to capture the waves reflected from the bottom of the mirror (it took about $10 \mu$ s for the longitudinal wave to make a round trip in the mirror). This gate length was confirmed to be sufficient for the present specimen as the amplitude of arrival waves to be measured dropped to the noise level in at most $6 \mu$ s after the first arrival wave. The received signals were digitized at the sampling frequency of $100 \mathrm{MHz}$ and averaged 100 times. The measured waveforms were then zero padded to contain 4096 points (resulting frequency increment was $0.02 \mathrm{MHz}$ ), Fourier transformed without windowing, and normalized by the spectrum of the reference wave measured without the specimen so as to compute the transmission coefficients.

The variation of the obtained transmission coefficient with the frequency and the angle of incidence for three types of planes of propagation $\left(\varphi=0^{\circ}\right.$ corresponds to the plane whose normal coincides with the fiber direction) is depicted in Fig. 11 for a finite frequency range of 5 to $15 \mathrm{MHz}$ due to the limited bandwidth of the transducer. It is noted that obtained by this measurement is the squared amplitude transmission coefficient since the doubly transmitted wave is detected. The theoretical results calculated by the stiffness matrix method are also shown in Fig. 11 for comparison, where the properties in Table I including the imaginary parts of ply stiffness are used with the interlayer interfacial stiffnesses $K_{\mathrm{N}}=3.0 \mathrm{MPa} / \mathrm{nm}$ and $K_{\mathrm{T}}=0.8 \mathrm{MPa} / \mathrm{nm}$. These parameters are chosen so that the theoretical transmission coefficients fit the experimental ones in Figs. 11(a)-11(c) as

\section{Experiment}

(a) $\varphi=0^{\circ}$

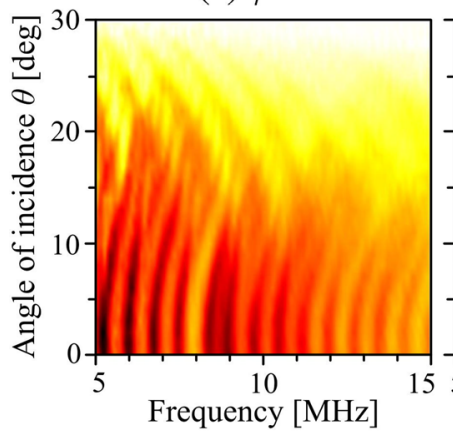

Frequency $[\mathrm{MHz}]$

(d) $\varphi=0^{\circ}$

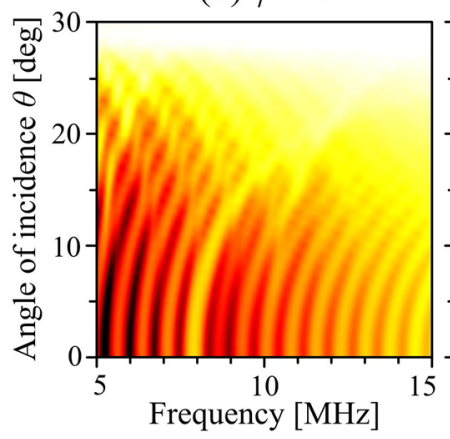

(b) $\varphi=45^{\circ}$

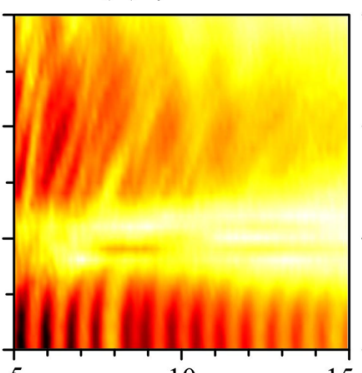

10

Theory

(e) $\varphi=45^{\circ}$

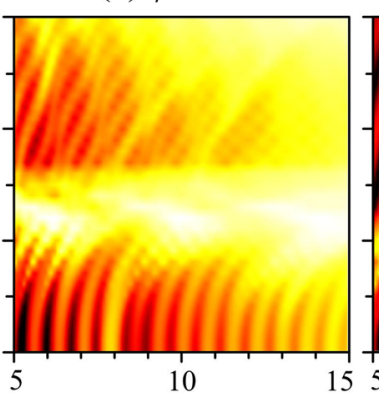

(c) $\varphi=90^{\circ}$

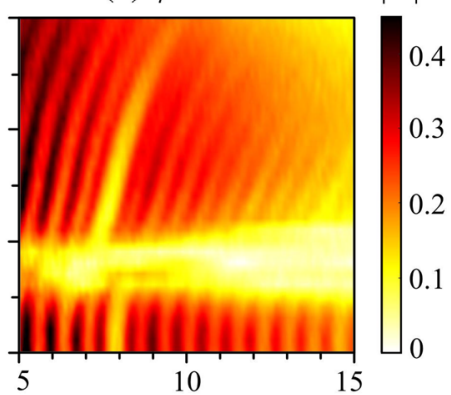

(f) $\varphi=90^{\circ}$

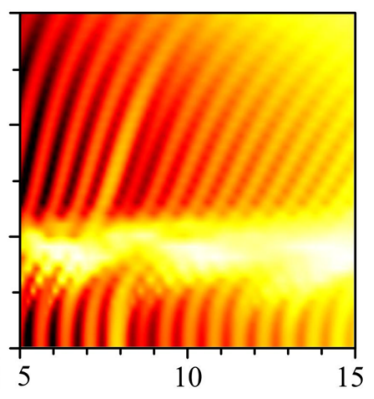

FIG. 11. (Color online) Experimental and theoretical squared amplitude transmission coefficients of an 11-ply carbon-epoxy unidirectional composite laminate for various frequencies and angles of incidence with three different planes of propagation. 
closely as possible. The values for the components of complex ply stiffness and the interfacial normal stiffnesses are representative for the unidirectional carbon-fiber-reinforced composite. $^{31,40}$ Although there are no literatures available for the interfacial shear stiffness of composite laminates, the above-mentioned value appears reasonable from the comparison of interfacial stiffness ratio $K_{\mathrm{T}} / K_{\mathrm{N}}=0.27$ with that measured for a thin epoxy adhesive layer between two glass plates $0.23 .{ }^{25}$

The low-transmission zones seen over the wide frequency ranges at around $\theta=30^{\circ}$ in Fig. $11(\mathrm{a}), \theta=10^{\circ}$ in Fig. 11(b), and $\theta=8^{\circ}$ in Fig. 11(c) are due to the critical angle of the quasi-longitudinal wave, while those seen in the limited frequency ranges such as at about $8 \mathrm{MHz}$ in Fig. 11(c) are due to the Bragg reflection. It is found that these features are well reproduced by the theory. It should be noted that the latter feature cannot be described unless the influence of interlayer interfaces is incorporated. Besides the locations of these low-transmission zones, fine oscillatory patterns of the amplitude transmission coefficient in the plane of frequency and incident angle are also reproduced by the theory with the interfacial stiffness parameters as identified above.

\section{SUMMARY}

In this paper, the transmission characteristics of ultrasonic waves in unidirectional composite laminates with spring-type interlayer interfaces at oblique incidence have been analyzed theoretically by using the stiffness matrix method. It has been shown that the amplitude transmission coefficient is influenced by the frequency, the interlayer interfacial normal and shear stiffnesses, and the angle of incidence. The dispersion relation of the corresponding infinitely periodic structure has been calculated using Floquet's periodic condition and compared to the transmission coefficient of the finite layered structure. As a result, the ranges of the frequency and the interfacial stiffnesses where the Floquet waves lie in the band-gaps are in good agreement with the low-transmission zones of the finite layered case, indicating that the ultrasonic wave is prevented from transmitting through the laminate due to the Bragg reflection. The amplitude transmission coefficient for an 11-ply carbon-epoxy composite laminate with unidirectional stacking sequence has been experimentally obtained for various frequencies and angles of incidence. It has been shown that the observed low-transmission zones caused by the critical angle of quasi-longitudinal wave as well as the Bragg reflection are favorably compared with the theory.

The results obtained in this analysis can be helpful when evaluating the quality of the interlayer interfaces of composite laminates from the propagation characteristics of bulk waves. The present study has focused on the laminate with unidirectional lay-up where the reflection and transmission at the interlayer interfaces are due only to the finite interfacial stiffnesses. For other stacking sequences, e.g., angle-ply, cross-ply, or quasi-isotropic laminates, the mismatch of the acoustic impedances between neighboring plies also has an influence on the scattering at the interfaces as discussed in
Ref. 20. In such situations, more complicated generation behavior of band-gaps will be observed, which remain as issues for the future study.

\section{ACKNOWLEDGMENTS}

This work has been supported by JSPS KAKENHI Grant Nos. 25-1754 and 25289005.

\section{APPENDIX: DERIVATION OF EQ. (17)}

Assuming that the $x_{3}$ component of the Floquet wavenumber is given as

$$
\zeta=(2 m \pi+a+i b) / h_{\mathrm{u}},
$$

where $m$ is an integer and $-\pi<a \leq \pi$ and $b$ are real numbers, Eq. (15) can be rewritten as

$$
\cosh b \cos a-i \sinh b \sin a=\frac{\eta}{2} .
$$

The band structure of the Floquet waves can be divided into four cases according to $\eta$ as follows:

$$
\begin{aligned}
& \text { (a) }|\operatorname{Re}[\eta]|<2 \text { and } \operatorname{Im}[\eta]=0, \\
& \text { (b) }|\operatorname{Re}[\eta]|=2 \text { and } \operatorname{Im}[\eta]=0, \\
& \text { (c) }|\operatorname{Re}[\eta]|>2 \text { and } \operatorname{Im}[\eta]=0, \\
& \text { (d) } \operatorname{Im}[\eta] \neq 0 .
\end{aligned}
$$

In the case of (a), Eq. (A2) is reduced to

$$
\begin{aligned}
& a= \pm \cos ^{-1}\left(\frac{\operatorname{Re}[\eta]}{2}\right), \\
& b=0 .
\end{aligned}
$$

In Eq. (A7), the plus-minus sign represents the pair of Floquet waves propagating in the opposite $x_{3}$ direction. Since the Floquet wavenumber becomes real from Eq. (A8), this case represents the outside of the band-gaps (passing bands).

In the case of (b), Eq. (A2) is reduced to

$$
\begin{aligned}
& a= \begin{cases}0, & \text { if } \operatorname{Re}[\eta]=2 \\
\pi, & \text { if } \operatorname{Re}[\eta]=-2,\end{cases} \\
& b=0 .
\end{aligned}
$$

This one hence corresponds to the boundaries between the passing band and the single band-gap.

In the case of (c), Eq. (A2) is reduced to

$$
\begin{aligned}
& a= \begin{cases}0, & \text { if } \operatorname{Re}[\eta]>2 \\
\pi, & \text { if } \operatorname{Re}[\eta]<-2,\end{cases} \\
& b=\ln \left[\frac{|\operatorname{Re}[\eta]|}{2} \pm \sqrt{\left(\frac{\operatorname{Re}[\eta]}{2}\right)^{2}-1}\right] \neq 0 .
\end{aligned}
$$


Since $b \neq 0$, this case corresponds to the single band-gap and Eq. (17) for $\alpha=\beta$ can be obtained from Eqs. (A1) and (A11).

In the case of (d), the following relations are obtained from Eq. (A2)

$$
\begin{aligned}
& a \neq 0 \text { and } a \neq \pi, \\
& b \neq 0 .
\end{aligned}
$$

From the property shown by Braga and Herrmann ${ }^{34}$ that if $X$ is a solution of Eq. (15), so are its reciprocal $1 / X$ as well as its complex conjugate $X^{*}$, it can be shown that if $\eta$ is a solution of Eq. (16), so is its complex conjugate $\eta^{*}$. Note that the above property was originally proved for the case where all plies are perfectly bonded, but it still holds for the case where the spring-type interlayer interfaces are incorporated. If the $x_{3}$ component of the Floquet wavenumber corresponding to $\eta$ is denoted by $\zeta_{\alpha}$ and given by the right-hand side of Eq. (A1) as $\zeta_{\alpha}=(2 m \pi+a+i b) / h_{\mathrm{u}}$, then one corresponding to $\eta^{*}$ and for the Floquet wave propagating in the same $x_{3}$ direction $\zeta_{\beta}$ can be written from Eq. (A2) as

$$
\zeta_{\beta}=(2 l \pi-a+i b) / h_{\mathrm{u}}
$$

where $l$ is an integer. From Eqs. (A13) and (A14), this case corresponds to the double band-gap, and Eq. (17) for $\alpha \neq \beta$ can be obtained.

${ }^{1}$ S. I. Rokhlin, D. E. Chimenti, and P. B. Nagy, Physical Ultrasonics of Composites (Oxford University Press, New York, 2011), pp. 225-368.

${ }^{2}$ M. F. Markham, "Measurement of the elastic constants of fibre composites by ultrasonics," Composites 1, 145-149 (1970).

${ }^{3}$ R. E. Smith, "Ultrasonic elastic constants of carbon fibers and their composites," J. Appl. Phys. 43(6), 2555-2561 (1972).

${ }^{4}$ B. Castagnède, J. T. Jenkins, W. Sachse, and S. Baste, "Optimal determination of the elastic constants of composite materials from ultrasonic wave-speed measurements," J. Appl. Phys. 67(6), 2753-2761 (1990).

${ }^{5}$ E. P. Papadakis, T. Patton, Y.-M. Tsai, D. O. Thompson, and R. B. Thompson, "The elastic moduli of a thick composite as measured by ultrasonic bulk wave pulse velocity," J. Acoust. Soc. Am. 89(6), 2753-2757 (1991).

${ }^{6} \mathrm{C}$. Aristégui and S. Bastle, "Optimal recovery of the elasticity tensor of general anisotropic materials from ultrasonic velocity data," J. Acoust. Soc. Am. 101(2), 813-833 (1997).

${ }^{7}$ S. I. Rokhlin and W. Wang, "Double through-transmission bulk wave method for ultrasonic phase velocity measurement and determination of elastic constants of composite materials," J. Acoust. Soc. Am. 91(6), 3303-3312 (1992).

${ }^{8}$ Y. C. Chu and S. I. Rokhlin, "Comparative analysis of throughtransmission ultrasonic bulk wave methods for phase velocity measurements in anisotropic materials," J. Acoust. Soc. Am. 95(6), 3204-3212 (1994).

${ }^{9}$ Y. C. Chu and S. I. Rokhlin, "A method for determination of elastic constants of a unidirectional lamina from ultrasonic bulk velocity measurements on [0/90] cross-ply composites," J. Acoust. Soc. Am. 96(1), 342-352 (1994).

${ }^{10} \mathrm{~B}$. Hosten, "Stiffness matrix invariants to validate the characterization of composite materials with ultrasonic methods," Ultrasonics 30(6), 365-371 (1992).

${ }^{11}$ B. Hosten, D. A. Hutchins, and D. W. Schindel, "Measurement of elastic constants in composite materials using air-coupled ultrasonic bulk waves," J. Acoust. Soc. Am. 99(4), 2116-2123 (1996).

${ }^{12}$ B. Hosten, M. Deschamps, and B. R. Tittmann, "Inhomogeneous wave generation and propagation in lossy anisotropic solids. Application to the characterization of viscoelastic composite materials," J. Acoust. Soc. Am. 82(5), 1763-1770 (1987).
${ }^{13} \mathrm{~B}$. Hosten, "Reflection and transmission of acoustic plane waves on an immersed orthotropic and viscoelastic solid layer," J. Acoust. Soc. Am. 89(6), 2745-2752 (1991).

${ }^{14} \mathrm{~S}$. Baudouin and B. Hosten, "Immersion ultrasonic method to measure elastic constants and anisotropic attenuation in polymer-matrix and fiber-reinforced composite materials," Ultrasonics 34, 379-382 (1996).

${ }^{15}$ S. Baudouin and B. Hosten, "Comparison between prediction and measurement of viscoelastic moduli in composite materials versus temperature using ultrasonic immersion technique with oil," J. Acoust. Soc. Am. 102(6), 3450-3457 (1997).

${ }^{16} \mathrm{M}$. Castaings, B. Hosten, and T. Kundu, "Inversion of ultrasonic, planewave transmission data in composite plates to infer viscoelastic material properties," NDT\&E Int. 33, 377-392 (2000).

${ }^{17}$ D. E. Chimenti and D. Fei, "Scattering coefficient reconstruction in plates using focused acoustic beams," Int. J. Solids Struct. 39, 5495-5513 (2002).

${ }^{18}$ N. Sela, O. Ishai, and L. Banks-Sills, "The effect of adhesive thickness on interlaminar fracture toughness of interleaved CFRP specimens," Composites 20(3), 257-264 (1989).

${ }^{19}$ D. H. Grande, L. B. Ilcewicz, W. B. Avery, and W. D. Bascom, "Effects of intra- and inter-laminar resin content on the mechanical properties of toughened composite materials," in Proceedings of First NASA Advanced Composite Technology Conference (1991), Vol. 2, 455-475.

${ }^{20} \mathrm{~L}$. Wang and S. I. Rokhlin, "Ultrasonic wave interaction with multidirectional composites: Modeling and experiment," J. Acoust. Soc. Am. 114(5), 2582-2895 (2003).

${ }^{21}$ A. Pilarski and J. L. Rose, "A transverse-wave ultrasonic obliqueincidence technique for interfacial weakness detection in adhesive bonds," J. Appl. Phys. 63(2), 300-307 (1988).

${ }^{22}$ S. I. Rokhlin and Y. J. Wang, "Analysis of boundary conditions for elastic wave interaction with an interface between two solids," J. Acoust. Soc. Am. 89(2), 503-515 (1991).

${ }^{23}$ A. Boström and G. Wickham, "On the boundary conditions for ultrasonic transmission by partially closed cracks," J. Nondestruct. Eval. 10(4), 139-149 (1991).

${ }^{24}$ S. I. Rokhlin and W. Huang, "Ultrasonic wave interaction with a thin anisotropic layer between two anisotropic solids: Exact and asymptoticboundary-condition methods," J. Acoust. Soc. Am. 92(3), 1729-1742 (1992).

${ }^{25}$ P. Fraisse, F. Schmit, and A. Zarembowitch, "Ultrasonic inspection of very thin adhesive layers," J. Appl. Phys. 72(8), 3264-3271 (1992).

${ }^{26} \mathrm{M}$. V. Golub, "Propagation of elastic waves in layered composites with microdefect concentration zones and their simulation with spring boundary conditions," Acoust. Phys. 56(6), 848-855 (2010).

${ }^{27}$ M. V. Golub and A. Boström, "Spring boundary conditions and modeling of 2D wave propagation in composites with imperfect interfaces," in Proceedings of International Conference Days on Diffraction (2010), pp. 73-78.

${ }^{28}$ Y. Lu and J. D. Achenbach, "Effects of random deviations in interface properties on the propagation of ultrasound in thick composites," J. Acoust. Soc. Am. 90(5), 2576-2585 (1991).

${ }^{29} \mathrm{Y}$. Lu, "Effects of random fluctuations in ply mechanical properties on ultrasound propagation in a laminated solid layer," Ultrasonics 30(5), 289-295 (1992).

${ }^{30}$ Y. Ishii and S. Biwa, "Ultrasonic evaluation of interlayer interfacial stiffness of multilayered structures,” J. Appl. Phys. 111, 084907 (2012).

${ }^{31}$ Y. Ishii and S. Biwa, "Evaluation of interlayer interfacial stiffness and layer wave velocity of multilayered structures by ultrasonic spectroscopy," J. Acoust. Soc. Am. 136(1), 183-191 (2014).

${ }^{32} \mathrm{~L}$. Wang and S. I. Rokhlin, "Stable reformulation of transfer matrix method for wave propagation in layered anisotropic media," Ultrasonics 39, 413-424 (2001).

${ }^{33}$ S. I. Rokhlin and L. Wang, "Stable recursive algorithm for elastic wave propagation in layered anisotropic media: Stiffness matrix method," J. Acoust. Soc. Am. 112(3), 822-834 (2002).

${ }^{34}$ A. M. B. Braga and G. Herrmann, "Floquet waves in anisotropic periodically layered composites," J. Acoust. Soc. Am. 91(3), 1211-1227 (1992).

${ }^{35}$ C. Potel, J.-F. Belleval, and Y. Gargouri, "Floquet waves and classical plane waves in an anisotropic periodically multilayered medium: Application to the validity domain of homogenization," J. Acoust. Soc. Am. 97(5), 2815-2825 (1995). 
${ }^{36}$ L. Wang and S. I. Rokhlin, "Floquet wave homogenization of periodic anisotropic media," J. Acoust. Soc. Am. 112(1), 38-45 (2002).

${ }^{37} \mathrm{~W}$. T. Thomson, "Transmission of elastic waves through a stratified solid medium," J. Appl. Phys. 21, 89-93 (1950).

${ }^{38}$ N. A. Haskell, "The dispersion of surface waves on multilayered media," Bull. Seismol. Soc. Am. 43, 17-34 (1953).
${ }^{39} \mathrm{~A}$. H. Nayfeh, "The general problem of elastic wave propagation in multilayered anisotropic media," J. Acoust. Soc. Am. 89(4), 1521-1531 (1991).

${ }^{40} \mathrm{~B}$. Hosten and M. Castaings, "Transfer matrix of multilayered absorbing and anisotropic media. Measurements and simulations of ultrasonic wave propagation through composite materials," J. Acoust. Soc. Am. 94(3), 1488-1495 (1993). 